\title{
Auxiliary partial orthotopic liver transplantation for acute liver failure
}

\author{
M Shrivastav ${ }^{1,2}$, A Rammohan ${ }^{1,2}$, MS Reddy ${ }^{1,2}$, M Rela $^{1,2,3}$ \\ ${ }^{1}$ Institute of Liver Disease \& Transplantation, Gleneagles Global Health City, Chennai, India \\ ${ }^{2}$ Institute of Liver Disease \& Transplantation, Dr.Rela Institute \& Medical Centre, Bharat Institute \\ of Higher Education \& Research, Chennai, India \\ ${ }^{3}$ Institute of Liver Studies, King's College Hospital, London, UK \\ ABSTRACT \\ INTRODUCTION Auxiliary partial orthotopic liver transplantation (APOLT) in acute liver failure acts as a bridge to native liver \\ regeneration with potential for immunosuppression free survival. While technical concerns limit its universal acceptance, the \\ indications in acute liver failure also need to be examined for this procedure to ultimately succeed. \\ CASE HISTORY We present the case of an eight-month-old girl with cryptogenic acute liver failure who underwent APOLT. She \\ developed postoperative liver dysfunction, most likely owing to the persistence of the diseased native liver, ultimately leading to \\ an orthotopic retransplantation. She remains well on follow-up review. \\ CONCLUSIONS A tempered approach to selecting patients for APOLT (especially with regard to aetiology of acute liver failure) \\ makes it a safe and effective alternative to orthotopic liver transplantation.
}

\section{KEYWORDS}

Auxiliary partial orthotopic liver transplantation - Acute liver failure - Aetiology - Outcomes

Accepted 2 June 2018

CORRESPONDENCE TO

Ashwin Rammohan, E: ashwinrammohan@gmail.com

Auxiliary partial orthotopic liver transplantation (APOLT) is a technically complex procedure in which a segmental liver graft is implanted orthotopically following a native liver partial hepatectomy. ${ }^{1}$ Despite being considered an innovative technique, it was thought to be a controversial procedure owing to its technically challenging nature and substandard outcomes. ${ }^{1,2}$ APOLT is gradually gaining acceptance as a standard of care in acute liver failure (ALF), where it acts as a bridge to native liver regeneration with the possibility of immunosuppression free survival. Selection of patients for APOLT has been variably defined, depending on the timing of ALF, age and condition of the recipient. ${ }^{1-4}$ The aetiology for ALF plays an important role. However, in patients with cryptogenic ALF, this remains a grey area. ${ }^{5}$ Outcomes may be suboptimal and the inciting event along with the injured liver can cause persisting injury to the graft.

\section{Case history}

An eight-month-old girl presented with a week's duration of jaundice along with reduced alertness over the previous three days. She had been evaluated and diagnosed with ALF elsewhere. With worsening mentation, progressively increasing serum bilirubin (from $9.5 \mathrm{mg} / \mathrm{dl}$ to $30 \mathrm{mg} / \mathrm{dl}$ ), an unresolving international normalised ratio of over 6 after correction and no improvement in clinical condition following medical management, she was referred to our unit for a possible liver transplant.

The aetiological workup along with infection, immunological and metabolic screening tests were unremarkable. The patient was put on the supra-urgent list for a deceased donor liver transplant. Her mother came forward to donate and was found suitable following a thorough donor workup. Intraoperatively, the baby remained stable and the liver showed regenerative nodules. The decision was made to perform APOLT. She received a left lateral segment graft with a graft-to-recipient weight ratio of 3.5 (Fig 1). The surgery was uneventful, with a peak intraoperative lactate of $5.6 \mathrm{mg} / \mathrm{dl}$ and a decreasing trend following reperfusion. No portal flow modulation was performed.

The initial postoperative period was uneventful and the patient was transferred to the ward on day 5. On day 15, she had elevated aspartate transaminase (651iu/l, normal range: $0-40 \mathrm{iu} / \mathrm{l})$. Doppler imaging was unremarkable with preferential portal flow to the graft. Consequently, she underwent a liver biopsy, which was suggestive of lobular hepatitis in both the native and graft livers (Fig 2). Her bilirubin showed a progressively upward trend, increasing from $1.9 \mathrm{mg} / \mathrm{dl}$ to $19 \mathrm{mg} / \mathrm{dl}$. Over the next five days, her 


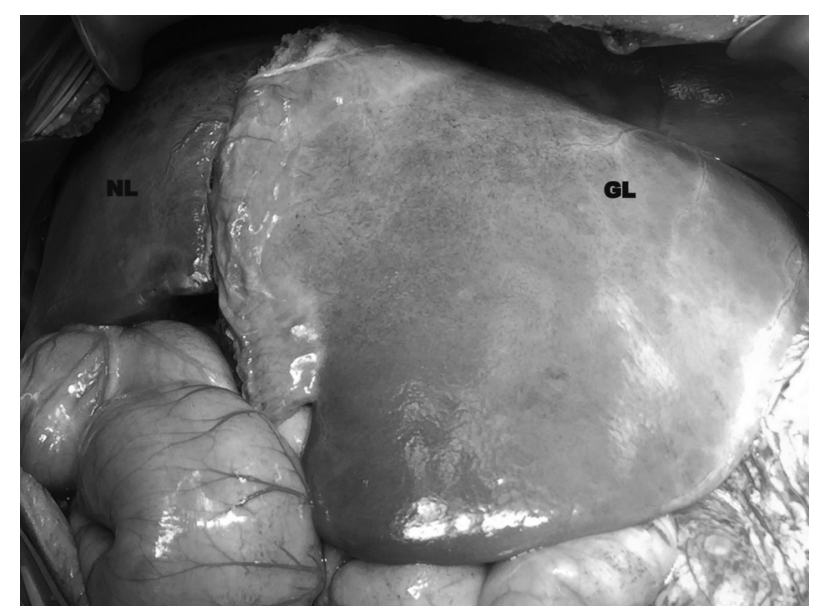

Figure 1 Intraoperative view of auxiliary partial orthotopic liver transplantation after implantation. $\mathrm{NL}=$ native liver; $\mathrm{GL}=$ graft liver

mentation deteriorated and her international normalised ratio remained over 10 . Given the risk of graft failure, she was considered for retransplantation. Her grandmother volunteered and was found fit to be a donor. The child underwent an orthotopic retransplantation after a native and graft hepatectomy. Seventeen days following her second transplant, she was discharged home. She remains well on follow-up review.

\section{Discussion}

Patients with ALF have a normal liver prior to the insult and a significant proportion of these patients can regenerate their hepatocyte mass if they survive long enough for regeneration to take place. ${ }^{1,4}$ APOLT is the only treatment option available at present that can act as a reliable bridge to liver regeneration so that patients may be spared lifelong immunosuppression.

Patient selection is important for APOLT. Success hinges primarily on the expected regenerative capacity of the native liver and the clinical condition of the patient at the time of transplantation. Children and younger adults have better liver regeneration than older adults. ${ }^{1,2,5,6}$ The type of graft is an important factor in patient recovery; compromising on volume and quality of the liver graft may confound outcomes. ${ }^{1-3,5}$ Our patient received a left lateral segment graft from her mother with an adequate graft-to-recipient weight ratio and is unlikely to have been adversely affected by the graft quality or volume.

The aetiology of ALF is also an important factor. While most criteria have been standardised, the aetiology most

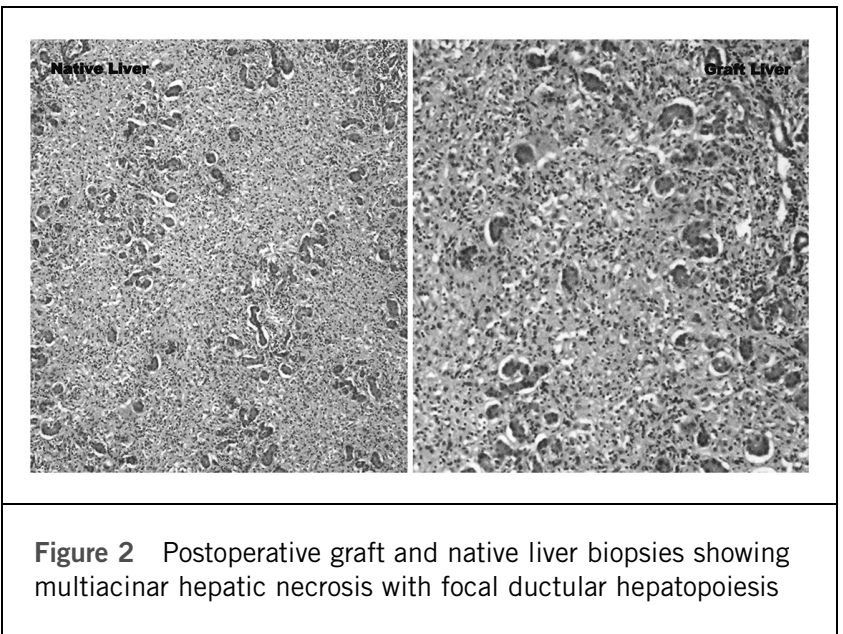

suitable for APOLT remains a relatively grey area, especially where rare causes like phosphorus poisoning are involved or in cases of cryptogenic ALF, where the primary inciting factor may persist, damaging the graft and compromising outcomes. ${ }^{1,5}$ As confirmed by the postoperative liver biopsy, this appears to have been the case with our patient. The second (orthotopic) liver transplant most likely removed the inciting factors for graft dysfunction, allowing for an uneventful recovery.

\section{Conclusions}

In the absence of clinical hepatocyte transplantation, APOLT provides the next best alternative for patients with ALF. Tiding over the crisis, it provides potential for immunosuppression withdrawal. A prudent approach to patient selection for APOLT (especially in terms of the aetiology of ALF) is an important factor in ensuring excellent outcomes.

\section{References}

1. Rela M, Kaliamoorthy I, Reddy MS. Current status of auxiliary partial orthotopic liver transplantation for acute liver failure. Liver Transp/2016; 22: 1,2651,274 .

2. van Hoek $\mathrm{B}$, de Boer $\mathrm{J}$, Boudjema $\mathrm{K}$ et al. Auxiliary versus orthotopic liver transplantation for acute liver failure. J Hepatol 1999; 30: 699-705.

3. Germani G, Theocharidou E, Adam R et al. Liver transplantation for acute liver failure in Europe: outcomes over 20 years from the ELTR database. $J$ Hepatol 2012; 57: 288-296.

4. Quaglia A, Portmann BC, Knisely AS et al. Auxiliary transplantation for acute liver failure: histopathological study of native liver regeneration. Liver Transp/ 2008; 14: 1,437-1,448

5. Azoulay D, Samuel D, Ichai P et al. Auxiliary partial orthotopic versus standard orthotopic whole liver transplantation for acute liver failure: a reappraisal from a single center by a case-control study. Ann Surg 2001; 234: 723-731.

6. Faraj W, Dar F, Bartlett A et al. Auxiliary liver transplantation for acute liver failure in children. Ann Surg 2010; 251: 351-356. 